\title{
ESTUDIO SISTÉMICO PARA DISMINUIR LA CONTAMINACIÓN POR RESIDUOS SÓLIDOS EN EL DISTRITO DE HUANCAYO
}

\author{
STUDY SYSTEMIC TO DIMINISH THE CONTAMINATION FOR SOLID \\ RESIDUALS IN THE DISTRICT OF HUANCAYO
}

\author{
Fidel Arauco Canturín
}

\section{RESUMEN}

Hay una necesidad imperante de facilitar como aporte a las autoridades para la mejor toma de decisiones, en el tratamiento adecuado de los residuos sólidos del Distrito de Huancayo, es por ello que se plantea el objetivo de construir un modelo sistémico de la generación y tratamiento de los residuos sólidos en la Ciudad de Huancayo mediante la metodología blanda de la dinámica de sistemas, este modelo permite identificar y determinar las principales variables que influyen en el tratamiento progresivo de los desechos orgánicos e inorgánicos, además que sirve como una herramienta de gestión para el manejo y manipulación eficiente de los mismos. Por lo que el tratamiento de los desechos o residuos sólidos se enfoca desde el pensamiento sistémico, para comprender y atacar este problema prioritario, debido a que los habitantes generan desechos como resultado de sus distintas actividades que realizan en su medio. La ciudad de Huancayo necesita resolver este problema que cada día es más nocivo y tiende a crecer la acumulación de desechos conforme la población va incrementándose, actualmente no hay un tratamiento previo ni planta de reciclaje, por lo que la contaminación sigue creciendo. El modelo es de enfoque cualitativo dominante, motivo por el cual la hipótesis general más que para probarse, ayuda a dar mayor sentido de entendimiento en el manejo adecuado de los residuos sólidos en nuestro medio, por otro lado se propone la interacción de acciones y políticas como educación ambiental y conciencia social para menguar este problema creciente. Mediante la simulación del modelo utilizando el software Stella 8.0 se obtuvieron escenarios favorables respecto a la solución del problema, y que por tanto se vería favorecida la población en tres aspectos: baja contaminación, recalentamiento local y disminución de personas enfermas por contaminación de desechos.

Palabras clave: Pensamiento sistémico, residuos sólidos, dinámica de sistemas.

\section{ABSTRACT}

There is a prevailing necessity to facilitate like contribution to the authorities for the best taking of decisions, in the appropriate treatment of the solid residuals of the District of Huancayo, is for it that thinks about the objective of building a systemic model of the generation and treatment of the solid residuals in the City of Huancayo by means of the soft methodology of the dynamics of systems, this model it allows to identify and to also determine the main variables that influence in the progressive treatment of the organic and inorganic waste, that it serves like an administration tool for the handling and efficient manipulation of the same ones. For what the treatment of the waste or solid residuals are focused from the systemic thought, to understand and to attack this high-priority problem, because the inhabitants generate waste as a result of their different activities that you/they carry out in their means. The city of Huancayo needs to solve this problem that every day is more noxious and it spreads to grow the according accumulation of waste the population he/she goes being increased, at the moment there are not a previous treatment neither recycle plant, for what the contamination continues growing. The pattern is of dominant qualitative focus, I motivate for the one which the general hypothesis more than to be proven, he/she helps to give bigger understanding sense in the appropriate handling of the solid residuals in our means, on the other hand he/she intends the interaction of actions and political as environmental education and social conscience to decrease this growing problem. By means of the simulation of the pattern using the software Stella 8.0 favourable scenarios was obtained regarding the solution of the problem, and that therefore it would be favoured the population in three aspects: low contamination, local heating and sick people's decrease for contamination of waste.

Key words: Systemic thought, solid residuals, dynamics of systems.

\footnotetext{
Facultad de Ingeniería de Sistemas. Universidad Nacional del Centro del Perú.
} 


\section{INTRODUCCIÓN}

El problema de la recolección, almacenamiento y disposición final de los desechos es responsabilidad de toda la población, en los diferentes niveles de organización de la Municipalidad Provincial de Huancayo, desde el gobierno local hasta los hogares; que para realizar el adecuado tratamiento de los desechos orgánicos e inorgánicos, está en función del comportamiento y actitud del individuo y de la sociedad, generado por su conciencia social y el grado de educación que posea.

Al utilizar la metodología blanda de la dinámica de sistemas para la solución del problema del manejo de los residuos sólidos en la Ciudad de Huancayo, se propone entre otros aspectos, establecer alternativas de solución de los desechos de manera sistémica (ver el conjunto holísticamente, causas, efectos y consecuencias en el sistema en estudio) y contribuye a proponer estrategias para el manejo adecuado de los desechos sólidos, contribuyendo para que el comportamiento frente a este problema sea más eficiente por parte de los habitantes del distrito de Huancayo.

Para muestra, la comuna provincial de Concepción, ha elaborado el proyecto Centro Ecoturístico y de protección ambiental Santa Cruz (CEPASC) con la única finalidad de dar un tratamiento y disposición adecuada a la basura, eliminando el foco de infección y contaminación ambiental y así promover la educación ambiental y ecológica para la protección ambiental y evitar el desequilibrio ecológico; formar el factor social y generar capacidades locales, para definir y emprender el camino hacia el desarrollo sustentable. El trabajo propone un tratamiento integral al problema de los desechos sólidos generados en la Ciudad de Huancayo, que hace mucho tiempo se viene discutiendo en nuestro medio y que las autoridades de turno no dan solución a este problema.

\section{MATERIAL Y MÉTODOS}

El modelamiento del sistema de la generación de residuos sólidos se realizó utilizando el Método de la Dinámica de Sistemas, abstrayéndose el modelo a partir de lo que se realiza en cuanto a la generación y recolección de lo residuos sólidos de la Metrópoli de Huancayo; para la muestra se tomó cien viviendas, obteniendo información primaria respecto a la cantidad de residuos sólidos generados por persona y el porcentaje de los tipos de residuos sólidos, así como las causas de ella; la información secundaria respecto a la población y el incremento para Huancayo se obtuvo del INEI para el año 2006.
El sistema solucionador del problema o analista (SSP) y el sistema contenedor del problema (SCP) se involucran en la situación problema, donde el analista actúa como sensor humano para diagnosticar, describir y obtener información resaltante respecto al manejo de los desechos, como también ayuda a descubrir posibles candidatos a problemas. Las instituciones que apoyaron con información relacionado a la contaminación por residuos sólidos en el distrito de Huancayo fueron: La gerencia de salud y medio ambiente (GSMA) de la Municipalidad Provincial de Huancayo (MPH), la oficina departamental del INEI, el diario Primicia de Huancayo, el plan integral de gestión ambiental de residuos sólidos (PIGARS), los estudios efectuados en el Centro Ecoturístico de Protección Ambiental Santa Cruz (CEPASC) de la ciudad de Concepción, el V Congreso Peruano de Ecología, Ambiente y Desarrollo Sostenible "JAVIER PULGAR VIDAL" desarrollado en la Ciudad de Concepción del 12 al 15 de Mayo de 2004. En cuanto a los materiales de trabajo para el procesamiento para el procesamiento de la información se utilizó el software estadístico SPSS y el Stella versión 8.0.

\section{RESULTADOS}

El estudio dio como resultado cinco sistemas, siendo los siguientes, los que se describen:

Sistema uno de actividad humana como parte del sistema de dirección, debe llevar en adelante la política para el tratamiento de los residuos sólidos, integrado por el Consejo Municipal (Comisión de Regidores) y la Alcaldía; quienes proponen las siguientes alternativas de solución: Invertir en la construcción de la planta de tratamiento de residuos sólidos en un lugar adecuado, realizar cultivos de forestación para controlar la contaminación ambiental local, contar con profesionales sociólogos, psicólogos, educadores y participación de la iglesia para enfrentar los malos hábitos en la población con respecto al manejo de los desechos.

Sistema dos de actividad humana como parte del sistema de gestión, debe llevar el informe de investigación para tratar los residuos sólidos en nuestro medio; integrada por la gerencia General y la Dirección de Medio Ambiente y Salud de la Municipalidad Provincial de Huancayo, la que propone las estrategias de descentralizar el poder, ser más flexible, delegar funciones, tomar decisiones, consultando al entorno e interno para que existe adaptación de aprendizaje y cambio deseado. Realizar ascensos, rotación en función a evaluación psicológica, académico, profesionalismo para afrontar la problemática en nuestro medio, para tratar los desechos sólidos en 
la ciudad de Huancayo, Identificar fuentes de financiamiento internacional, ingreso propio mediante la tecnología de reciclaje de residuos sólidos, trabajo en equipo con las instituciones del medio para erradicar medios informativos con contenido social negativo.

Sistema tres de actividad humana, como parte del sistema de gestión responsable de la estabilidad interna de la organización. Para lograr el tratamiento adecuado de los residuos sólidos en nuestro medio de acuerdo al informe de investigación, conformada por la Gerencia de Salud y Medio Ambiente (GSMA), Gerencia de Desarrollo Humano(GDH), Gerencia de Comercialización y Ferias(GCF); las políticas que plantea este sistema son: evaluar la información del sistema dos y tres para una mejor toma de decisiones, solicitar presupuesto adecuado para desarrollar tecnología de reciclaje, para el tratamiento de los residuos sólidos, autónomo y no esperar la voluntad política para desarrollar el tratamiento de los residuos sólidos en el distrito de Huancayo y solicitar mayores recursos en educación para un modelo de gestión ambiental, mientras que las alternativas de solución que plantea se encuadran en una visión compartida, para combatir los desechos donde cada vez más y más va desarrollándose epidemias y enfermedades, realizar talleres de innovación tecnológica de cómo influyen el aspecto económico, tiempo y calidad en el tratamiento de los residuos sólidos, influir en el cambio de mentalidad positiva en la población mediante charlas en forma gratuita, realización de congresos, seminarios sobre la conservación, innovación y la preservación de los recursos naturales, sociales, culturales y ambientales frecuentemente para atacar el problema de los desechos.

Sistema dos de actividad humana, como parte del sistema de monitoreo y control, responsable de proveer información solicitada al sistema tres (comisión del sistema tres).

Este sistema debe llevar acabo reuniones capacitaciones, charlas, con apoyo de sociólogos, psicólogos, ambientalistas e ingeniero químico para afrontar el problema de los desechos sólidos.
Tabla 1. Producción de tipos de residuos sólidos en \%.

\begin{tabular}{lc}
\hline Tipo de residuo sólido & Porcentaje (\%) \\
\hline Papely cartones & 6 \\
Plásticos & 6 \\
Materia orgánica & 65 \\
Metal & 3 \\
Huesos & 1 \\
Textiles & 3 \\
Vidrio & 2 \\
Madera & 1 \\
\hline
\end{tabular}

Fuente: Digesa, Lima, 2003.

Sistema uno de actividad humana viable o autocontrol, como parte del sistema de monitoreo y control responsable de las tareas operativas como el tratamiento de los residuos sólidos hacia el

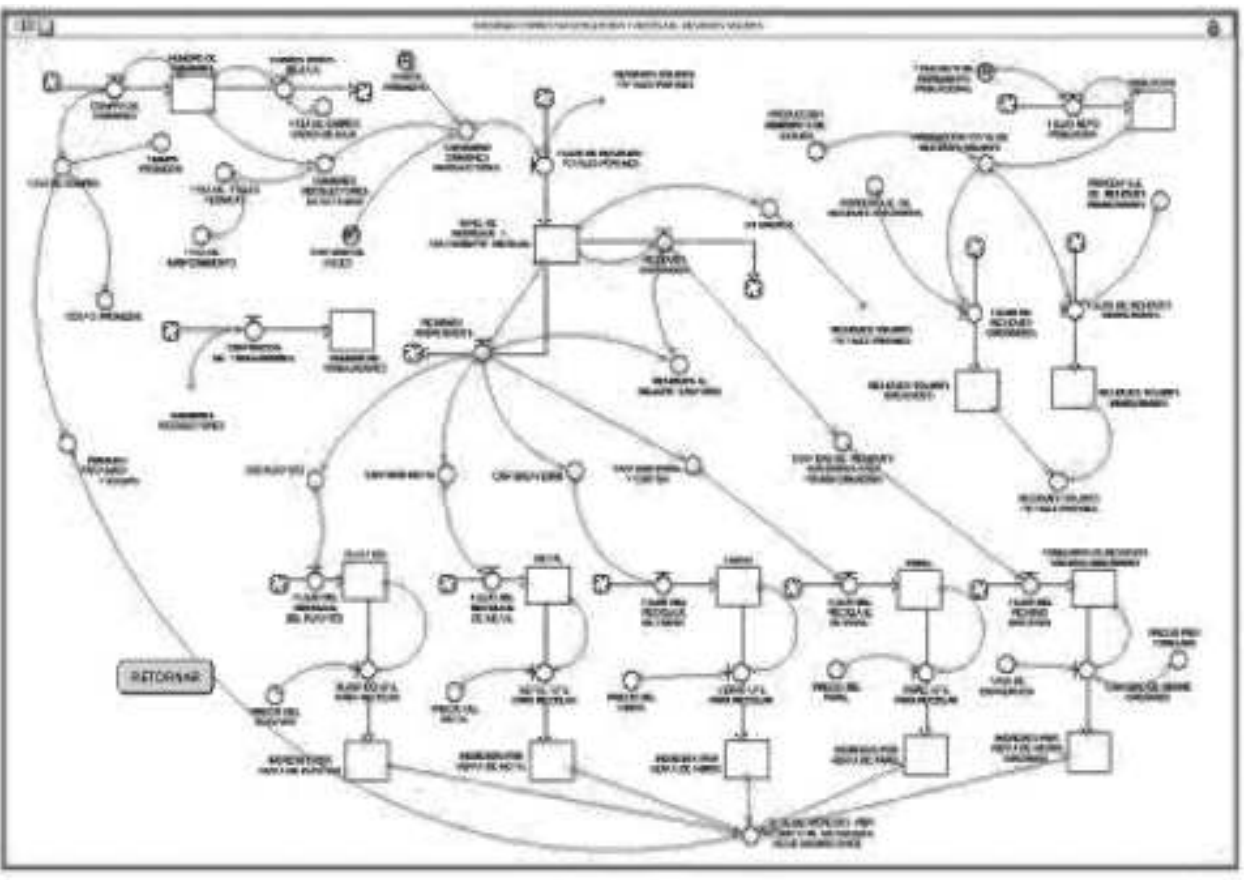

\section{Figura 1. Diagrama Forrester del sistema de producción y manejo de los residuos sólidos.}

Fuente: Elaboración propia.

sistema tres, la conforman la junta vecinal, sindicato de comerciantes e industriales, capataz general de limpieza publica, sindicato de mercados y mercadillos; ellos plantean las siguientes políticas: mejorar plan de comunicación para interactuar de manera eficiente con las autoridades, coordinar con las instituciones de su medio y otros para ser eficientes en la generación, recolección y almacenamiento de residuos sólidos, solicitar apoyo de profesionales en sistémica, ambientalistas, sociólogos para estar acorde a las tendencias mundiales, teniendo en cuenta la responsabilidad social; las alternativas de solución: 
clasificar los residuos orgánico e inorgánico desde los hogares y otros, clasificar los residuos orgánico e inorgánico según la calidad y grado de utilidad, obtener subproductos inorgánicos útiles para la comercialización, confinar al relleno sanitario los residuos inorgánicos no reciclables, producir abono para la agricultura.

\section{DISCUSIÓN}

La metodología blanda de la dinámica de sistemas, es una metodología que ayuda a conocer el comportamiento de los sistemas sociales, tal como es el manejo de los residuos sólidos como un todo, permite plantear soluciones al problema mediante políticas y propuestas más adecuadas, de manera que el tratamiento de los residuos sólidos sea eficiente.

En el desarrollo del trabajo se emplea en su mayoría el enfoque cualitativo, y sirve para incrementar el conocimiento sobre el manejo de los residuos sólidos producidos en la Ciudad de Huancayo, de esta manera ayuda a dar mayor sentido de entendimiento en el tratamiento de los desechos orgánicos e inorgánicos mediante la metodología blanda de la dinámica de sistemas (MBDS).

El modelo dinámico es interpretado por el computador, mediante el modelo forrester que se desarrolla en el software Stella versión 8.0 para luego experimentar o simular obteniendo diversos escenarios al implantar las políticas en el modelo.

Experimentar en el sistema real el manejo adecuado de los residuos sólidos hubiera sido tedioso, difícil, costosísimo en dinero y tiempo, para finalmente conseguir o no conseguir las respuestas deseadas. Pero la simulación ayuda a observar como debe ser el comportamiento adecuado del manejo de los desechos en función a educación ambiental y conciencia social.

\section{REFERENCIAS BIBLIOGRAFICAS}

1. Johansen O. Introducción a la Teoría General de Sistemas. Editorial Limusa, México;1984.

2. Rodríguez R. La sistémica, los sistemas blandos y los sistemas de información. Lima; 1994

3. López H, Sotaquira R. Pensamiento sistémico: diversidad en búsqueda de unidad. Edit. y de Publicaciones Universidad Industrial de Santander Bucaramanga, Colombia; 2001.

4. Senge P. La quinta disciplina. Como Impulsar el Aprendizaje en la Organización Inteligente. Edit., Juan Granica S.A., Barcelona, España; 1993.

5. Aracil J. Introducción a la Dinámica de Sistemas. Edit. Alianza: Madrid, España; 1983.

E-mail: fidelarauco12@yahoo.es 